\title{
Physical Investigations on Bias-Free, Photo-Induced Hall Sensors Based on Pt/GaAs and Pt/Si Schottky Junctions
}

\author{
Xiaolei Wang ${ }^{1, *} \mathbb{B}$, Xupeng Sun ${ }^{1}$, Shuainan Cui ${ }^{1}$, Qianqian Yang ${ }^{1}$, Tianrui Zhai ${ }^{1}{ }^{1}$, Jinliang Zhao ${ }^{1}$, \\ Jinxiang Deng ${ }^{1}$ and Antonio Ruotolo ${ }^{2}$ (D) \\ 1 College of Physics and Optoelectronics, Faculty of Science, Beijing University of Technology, \\ Beijing 100124, China; Sunxp@emails.bjut.edu.cn (X.S.); cuisn202006096@emails.bjut.edu.cn (S.C.); \\ yangqianqian@bjut.edu.cn (Q.Y.); trzhai@bjut.edu.cn (T.Z.); zhaojinliang@bjut.edu.cn (J.Z.); \\ jdeng@bjut.edu.cn (J.D.) \\ 2 Department of Natural Sciences, Florida Polytechnic University, 4700 Research Way, \\ Lakeland, FL 33805, USA; aruotolo@floridapoly.edu \\ * Correspondence: xiaoleiwang@bjut.edu.cn
}

Citation: Wang, X.; Sun, X.; Cui, S.; Yang, Q.; Zhai, T.; Zhao, J.; Deng, J.; Ruotolo, A. Physical Investigations on Bias-Free, Photo-Induced Hall Sensors Based on $\mathrm{Pt} / \mathrm{GaAs}$ and $\mathrm{Pt} / \mathrm{Si}$ Schottky Junctions. Sensors 2021, 21, 3009. https://doi.org/10.3390/ s21093009

Academic Editor: Raffaele Velotta

Received: 23 February 2021

Accepted: 23 April 2021

Published: 25 April 2021

Publisher's Note: MDPI stays neutral with regard to jurisdictional claims in published maps and institutional affiliations.

Copyright: (c) 2021 by the authors. Licensee MDPI, Basel, Switzerland. This article is an open access article distributed under the terms and conditions of the Creative Commons Attribution (CC BY) license (https:// creativecommons.org/licenses/by/ $4.0 /)$.

\begin{abstract}
Hall-effect in semiconductors has wide applications for magnetic field sensing. Yet, a standard Hall sensor retains two problems: its linearity is affected by the non-uniformity of the current distribution; the sensitivity is bias-dependent, with linearity decreasing with increasing bias current. In order to improve the performance, we here propose a novel structure which realizes bias-free, photo-induced Hall sensors. The system consists of a semi-transparent metal $\mathrm{Pt}$ and a semiconductor Si or GaAs to form a Schottky contact. We systematically compared the photo-induced Schottky behaviors and Hall effects without net current flowing, depending on various magnetic fields, light intensities and wavelengths of $\mathrm{Pt} / \mathrm{GaAs}$ and $\mathrm{Pt} / \mathrm{Si}$ junctions. The electrical characteristics of the Schottky photo-diodes were fitted to obtain the barrier height as a function of light intensity. We show that the open-circuit Hall voltage of $\mathrm{Pt} / \mathrm{GaAs}$ junction is orders of magnitude lower than that of $\mathrm{Pt} / \mathrm{Si}$, and the barrier height of GaAs is smaller. It should be attributed to the surface states in GaAs which block the carrier drifting. This work not only realizes the physical investigations of photoinduced Hall effects in Pt/GaAs and Pt/Si Schottky junctions, but also opens a new pathway for bias-free magnetic sensing with high linearity and sensitivity comparing to commercial Hall-sensors.
\end{abstract}

Keywords: Schottky junction; barrier height; photo-induced Hall effect; magnetic sensor

\section{Introduction}

The Hall effect in semiconductors has been used for more than one century to detect the intensity of magnetic fields [1]. Although magneto-resistive sensors in digital systems have been developed in recent years, semiconductor Hall sensors still retain irreplaceable in analog applications because of their two specific features: (1) they are intrinsically linear, and do not need magnetic materials; (2) their sensitivity is directly proportional to the bias current [2-7]. Yet, commercial Hall sensors have to operate by using a very low bias current, because linearity decreases with increasing bias-current [8]. On the contrary, the Hall effect also exists in metals. The low resistance of metals could increase uniformity of the current density and reduce dependence of linearity on bias current $[9,10]$. However, the transverse voltage is too small to have any practical application [11].

Lateral effect, Schottky photo-diodes are commonly used as magnetic- and position sensing- detectors [12]. Photo-excitation of carriers in a semiconductor can increase the Hallvoltage without increasing bias current. The photo-induced Hall effect in a Schottky diode, consisting of "metal/semiconductor" hetero-structure, includes the following physical processes: photo-generating in semiconductor, carriers separating and drifting across the interface, then diffusing in metal driven by a magnetic force [13]. A photon with 
energy larger than the band-gap of semiconductors could be absorbed during the photogeneration. It could transfer an electron from valence band to conduction band, or a hole from conduction band to valence band. Separation of carriers is achieved through the space charge region of a Schottky barrier, which relies on the built-in electric field. The carriers in semiconductor are injected into metal at a very high velocity, much faster than diffusion [13]. In this case, if a magnetic field is applied in the plane of a Schottky diode, it can generate large Lorentz forces and produce a transverse, open-circuit voltage at the metal edges that is proportional to the field, as well as light intensity. For a traditional lateral photo-diode, light is photo-converted into the increasing of an existed electrical current in a standard device [14-17]. Specifically, it consist of an extended p-type/intrinsic/ntype (p-i-n) semiconductor tri-layer with a bottom cathode electrode and four top lateral anodes. The p-type layer is thin enough to allow penetrating of light and resistive enough to avoid flowing of in-plane current through low doping concentration of acceptors. On the other side, the n-type layer is highly doped with donors in order to easily collect the current through the cathode. Therefore, the traditional lateral-effect photo-diode is a close-circuit, in which light beam causes a change of current flowing between the anodes and the cathode. However, current state-of-the-art, non-uniformity of the in-plane current distribution compromises linearity in these kinds of sensors. In contrast, in our device, light only reduces the built-in potential of the Schottky-barrier, with no net current flowing. This allows our sensor to operate in open-circuit conditions to recover linearity without increasing cost.

It has been recently reported $[13,18-20]$ that a giant photo-Hall effect is induced in a novel device structure as mentioned above. A typical system consists of an ultra-thin $\mathrm{Pt}$ film that forms a Schottky contact to intrinsic Si. Compared to a standard Hall sensor, in-plane current is replaced by light, the magnetic field is applied in plane instead of perpendicular. It shows high linearity and sensitivity which are comparable to commercial Hall-sensors. Moreover, there is no net current in the circuit, so its performances are not affected by bias current.

\section{Materials and Methods}

It is necessary to engineer and optimize this novel Hall-sensor based on photo-induced Hall effect. Various Schottky bilayers could be explored to fabricate bias-free, opticallytunable Hall sensors [21]. The possibility to optimize the photo-Hall effect should be investigated by replacing the normal metal or the semiconductor. In order to form a highly rectifying Schottky contact, the metal is usually $\mathrm{Au}$ or $\mathrm{Pt}$, which have work functions of $\phi \mathrm{Au}=5.5 \mathrm{eV}$ and $\phi \mathrm{Pt}=5.9 \mathrm{eV}$, respectively [22]. So Pt should be the best choice in this system. Instead, replacing semiconductor $\mathrm{Si}$ with a direct band-gap semiconductor, such as GaAs [23], may help to understand the physical mechanism and even increase sensitivity of the bias-free, photo-induced Hall sensors.

In order to verify the above idea, we carried out systematical experiments on $\mathrm{Pt} / \mathrm{GaAs}$ and $\mathrm{Pt} / \mathrm{Si}$ junctions. The intrinsic $\mathrm{GaAs}$ and $\mathrm{Si}$ wafers used in this work were single-side polished, (100)-oriented, with resistivity greater than $10,000 \Omega \cdot \mathrm{cm}$. The carrier concentration of $\mathrm{Si}$ is $1.5 \times 10^{10} \mathrm{~cm}^{-3}$, while the carrier concentration of GaAs is $1.8 \times 10^{6} \mathrm{~cm}^{-3}$. The semi-transparent $\mathrm{Pt}$ films of $\sim 3 \mathrm{~nm}$ were grown by high vacuum sputtering from a $99.99 \%$ pure $\mathrm{Pt}$ target, with $\mathrm{DC}$ power supply $50 \mathrm{~W}$. The base pressure was $2 \times 10^{-6} \mathrm{~Pa}$ and the process gas (Ar) pressure was $0.2 \mathrm{~Pa}$. The growth rate was calculated to be $0.15 \mathrm{~nm} / \mathrm{s}$ and $20 \mathrm{~s}$ were used for our deposition. The ultra-thin Pt films were grown on both entire 2-inch $\mathrm{GaAs}$ and $\mathrm{Si}$ wafers, and then were cut into chips for current-voltage (I-V) characterizations and photo-Hall measurements. Before the top Pt layer deposition, small part of GaAs or Si surface was partially masked in order to access the bottom electrode of a Schottky diode. The size of Pt film in a Schottky junction was kept to be $1 \times 1 \mathrm{~cm}^{2}$. Electrical contacts of the Schottky device were made by using silver paste to avoid the puncture of Pt thin film. The open-circuit Hall voltage was obtained by using a Keithley nanovoltmeter under an electromagnet. 


\section{Results}

\subsection{Schottky Rectifying Behaviors}

Figure 1a shows the representation for our typical Schottky device of $\mathrm{Pt} / \mathrm{GaAs}$ or Pt $/ \mathrm{Si}$ junction in this work. Since the intrinsic wafer is highly resistive, the electrical setup could reveal the existence of a Schottky barrier at the interface. A significant series resistance $\left(R_{S}\right)$ is added by the GaAs or Si that can be subtracted to estimate the barrier height. Figure $2 \mathrm{~b}$ shows that the light source is a commercial optical fiber illuminator emitting non-polarized, white light with a wavelength spectrum ranging from $\lambda=400 \mathrm{~nm}$ (violet) to $\lambda=800 \mathrm{~nm}$ (red), with maximum power $150 \mathrm{~W}$. Light was uniformly shed on the sample.

(a)

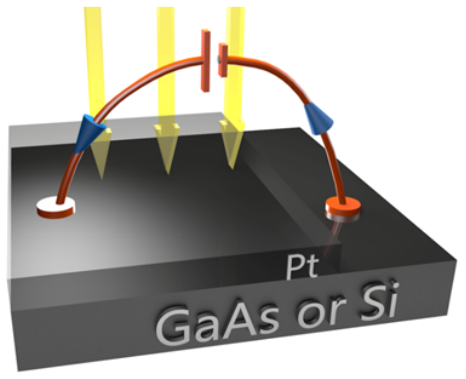

(c)

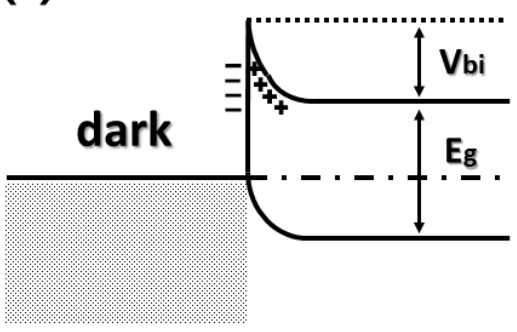

(b)

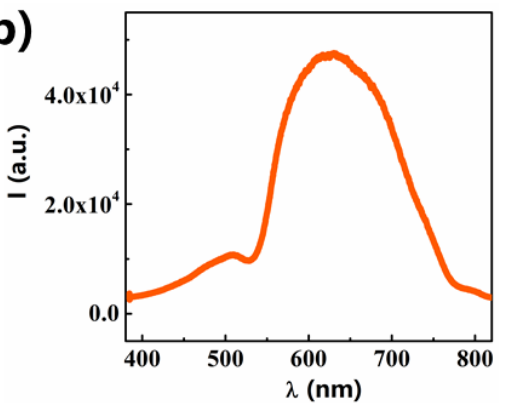

(d)

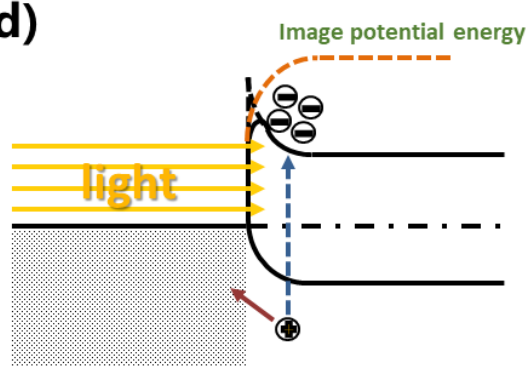

Figure 1. (a) The configuration for our typical Schottky device of $\mathrm{Pt} / \mathrm{GaAs}$ or $\mathrm{Pt} / \mathrm{Si}$. (b) The spectrum of the light we used, covering all visible range. (c) Representation of the equilibrium corresponding to the alignment of the Fermi levels for a Schottky junction. $V_{b i}$ is the theoretically calculated built-in potential. (d) Sketch of the photo-induced excitation of carriers and lowering of the barrier due to image forces.

Figure 1c,d show the physical origin of a Schottky photo-diode according to the theory of image forces [24]. If the metal has a work-function much higher than the semiconductor, a Schottky junction is formed at the interface [25]. Electrons move from the semiconductor to the metal, leaving uncompensated holes in the semiconductor. At equilibrium, the builtin potential $\mathrm{V}_{\mathrm{bi}}$ prevents additional carriers to migrate. This equilibrium corresponds to the alignment of the Fermi levels, as shown in Figure 1c. When the sample is illuminated, light can penetrate through the semi-transparent metal and electron-hole pairs are generated [26]. Electrons can readily flow from the metal to the semiconductor to re-establish equilibrium, but electrons can neither overcome the barrier nor flow through the intrinsic semiconductor. Yet, an additional electron in the space charge region results in lowering of the barrier height, which can be modeled by the theory of image forces [27]. Considering an electron trapped at a distance $\mathrm{z}$ from the interface, a positive mirror image charge is induced in the metal at a distance $-z$. A second electric field builds up, with an opposite sign as compared to the built-in potential. The image potential energy associated with this field is given by the following Equation (1) and decreases with the distance $\mathrm{z}$ from the interface. It results in a rounding of the net potential barrier as depicted in Figure $1 \mathrm{~d}$.

$$
\phi(z)=-\int_{x}^{\infty} E_{I}(z) d z=\frac{q}{16 \pi \varepsilon z}
$$


Despite the significant series resistance offered by the GaAs or Si substrate, both I-V characteristics are clearly rectifying in Figure 2a,b. The current of $\mathrm{Pt} / \mathrm{GaAs}$ junction is about one order of magnitude lower than that of $\mathrm{Pt} / \mathrm{Si}$, which may originate from the larger resistivity of GaAs. We show the influence of different light powers on Schottky behavior of both samples. Larger light intensity could excite more carriers in a semiconductor, so it increases the current of the Schottky junction and improve the rectifying behavior.

(a)

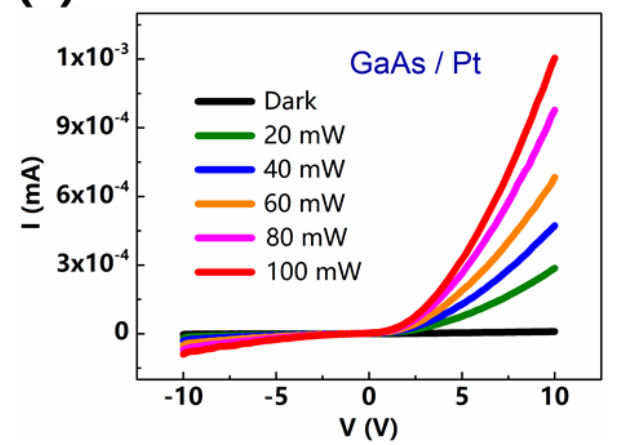

(b)

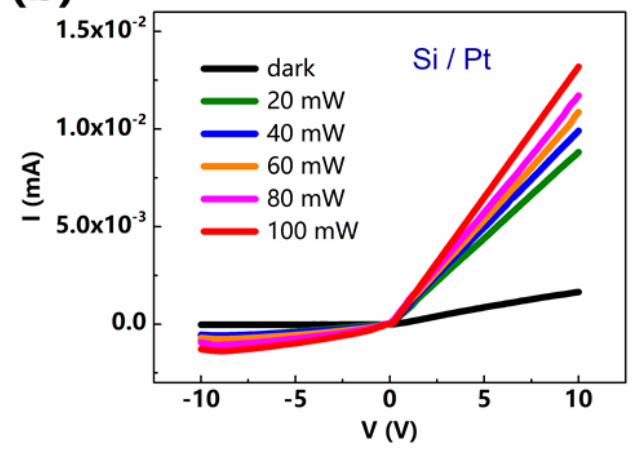

Figure 2. Current-voltage (I-V) characteristics of (a) Pt/GaAs and (b) Pt/Si bilayers in the dark and under different light intensities. Light intensities of $20 \mathrm{~mW}, 40 \mathrm{~mW}, 60 \mathrm{~mW}, 80 \mathrm{~mW}$ and $100 \mathrm{~mW}$ were detected on the sample surface.

\subsection{Photo-Induced Hall Effects}

For typical Hall effect in metals, a given current is injected along the x-direction and magnetic field is applied along the z-direction. Hall voltage $V_{H}$ is measured along $\mathrm{y}$-direction, as indicated in Figure $3 \mathrm{a} . V_{H}$ is inversely proportional to the carrier density $n$. As $n$ is very large in metals, $V_{H}$ is very small and difficult to be detected [28]. A novel photo-Hall sensor was devoted to increase the Hall effect in metals to magnitudes suitable for applications [29]. Figure $3 \mathrm{~b}$ shows the schematic representation of photoHall effect by injecting light-excited carriers at high velocity through the interface of a metal/semiconductor Schottky contact.

The light source was the same one as Figure $1 \mathrm{~b}$ and uniformly shed on the sample along the z-direction, shown as in Figure $3 b$, with the magnetic field applied along the $x$-direction and the open-circuit voltage measured along y-direction.

We engineered a similar system in Figure 3b, in which the Hall effect in Pt is greatly enhanced and dependence of linearity on bias current is suppressed. The device recovers tunable sensitivity, without compromising linearity, by replacing current bias with light bias. Light reaching the interface provides the force to drive carriers across the bilayer. A magnetic field applied in the junction plane exerts a magnetic force on the carriers diffusing in the metal, which accumulates on opposite sides, according to their sign. This carrier accumulation can be detected as a transverse, open-circuit voltage on the metal surface. $V_{H}$ is proportional to the field strength, as well as light intensity and wavelengths, as shown in Figures 4 and 5. At equilibrium, the net current is zero regardless light intensity. 
(a)

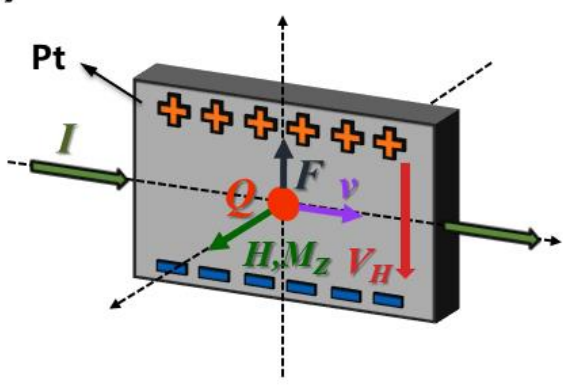

(c)

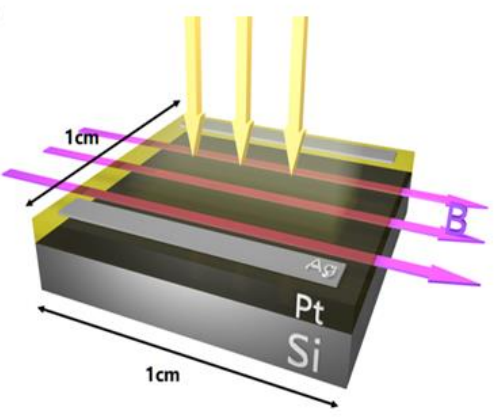

(b)

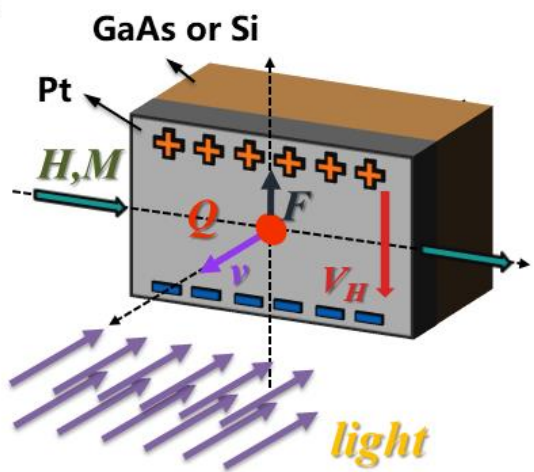

(d)

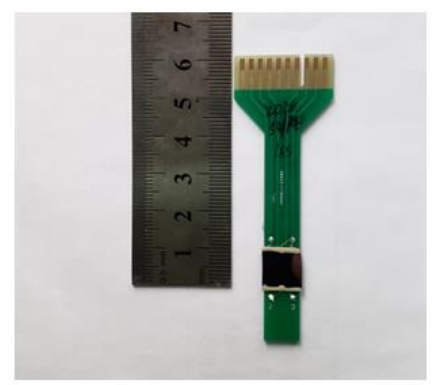

Figure 3. (a) Hall effect in a Pt film; (b) Photo-induced Hall effect in a Schottky photo-diode; (c) The configuration of our photo-Hall device under magnetic field; (d) The picture of our real device and sample holder for electrical connection.

(a)

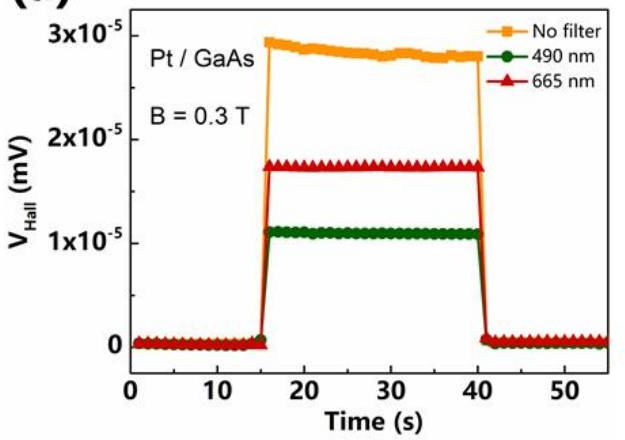

(c)

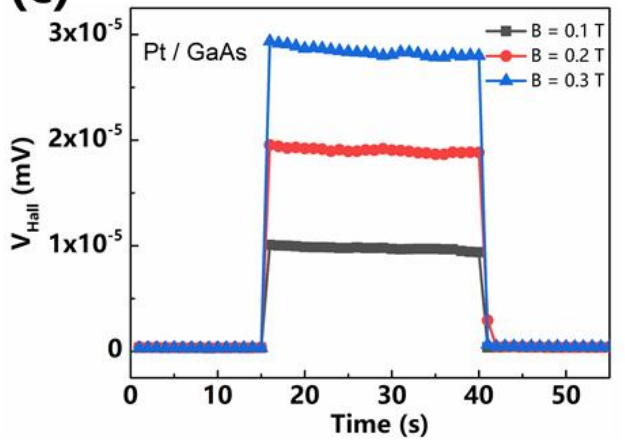

(b)

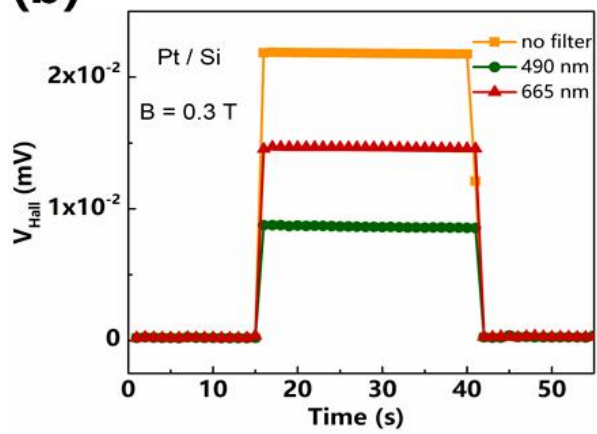

(d)

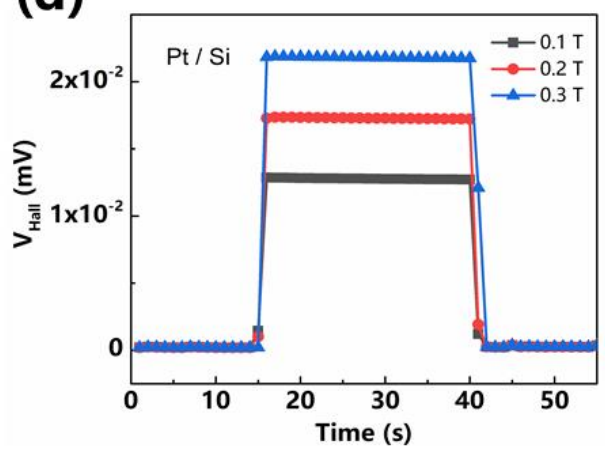

Figure 4. Photo-excitation of transverse, photo-induced Hall voltage vs. time elicited when exposing the (a) $\mathrm{Pt} / \mathrm{GaAs}$ and (b) $\mathrm{Pt} / \mathrm{Si}$ bilayers to light in different wavelength ranges as magnetic field $\mathrm{B}=0.3 \mathrm{~T}$. Similar signals were obtained under different magnetic fields $\mathrm{B}=0.1,0.2$, and $0.3 \mathrm{~T}$ for (c) $\mathrm{Pt} / \mathrm{GaAs}$ and (d) Pt/Si junctions under a constant while light. The power of light source in all the measurements was kept $\mathrm{P}=50 \mathrm{~mW}$. 
(a)

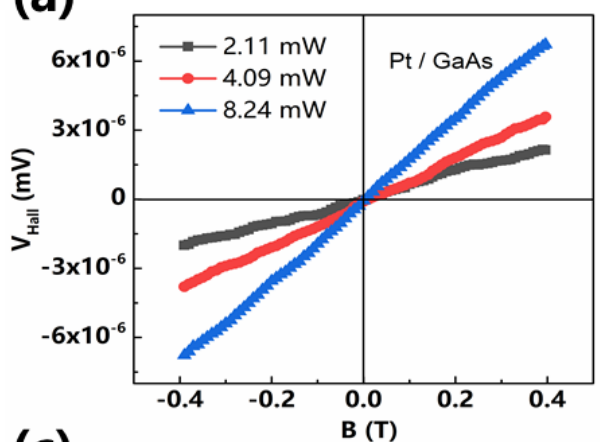

(c)

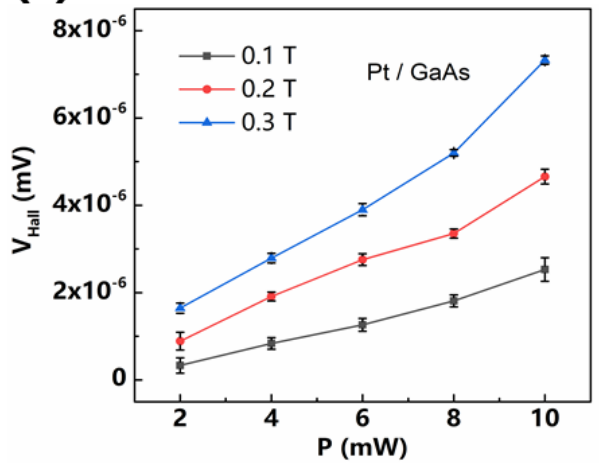

(b)

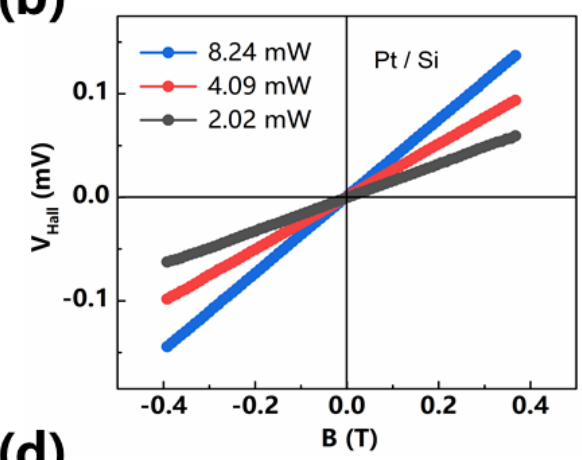

(d)

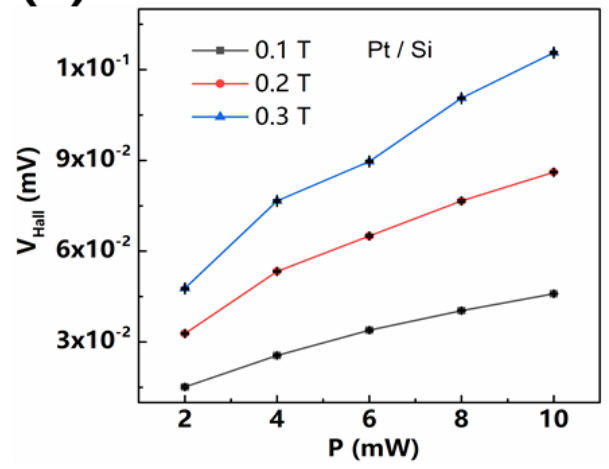

Figure 5. $V_{H^{-}}$at constant light intensities for (a) $\mathrm{Pt} / \mathrm{GaAs}$ and (b) $\mathrm{Pt} / \mathrm{Si}$. $V_{H^{-}} \mathrm{P}$ at constant magnetic fields of (c) Pt/GaAs and (d) Pt/Si with error bars.

Photo-induced Hall measurements were carried out by applying light in different wavelengths ranges achieved by filters and full spectrum of the lamp, labeled as "no filter", as magnetic field B $=0.3 \mathrm{~T}$ in Figure $4 \mathrm{a}, \mathrm{b}$. In the visible range, the photo-conversion increases from short wavelengths to longer ones. Different $V_{H}$ signals come from different photon-conversion energies. Noticeably, the open-circuit $V_{H}$ of $\mathrm{Pt} / \mathrm{GaAs}$ shows orders of magnitude lower than $V_{H}$ of $\mathrm{Pt} / \mathrm{Si}$, which is out of expected. Similar results were obtained in magnetic field dependent of $V_{H}$. As the field increases, so does the Lorentz force acting on the carriers and the photo-induced $V_{H}$, indicated in Figure 4c,d.

In Figure 5, this effect was also found to be linear with both magnetic field strength and light intensity. We show the detected $V_{H}$ as a function of the field strength under different light intensities in Figure $5 \mathrm{a}, \mathrm{b}$. The voltage was proportional to the magnetic field strength. In Figure $5 c, d, V_{H}$ increased linearly with light intensity for fixed values of magnetic field. Following the same law in Figure 4, the effect depending on magnetic field and light intensity of $\mathrm{Pt} / \mathrm{GaAs}$ shows orders of magnitude lower than $V_{H}$ of $\mathrm{Pt} / \mathrm{Si}$.

The comparison of the experimetal results on $\mathrm{Pt} / \mathrm{GaAs}$ and $\mathrm{Pt} / \mathrm{Si}$ permitted the deduction of surface state effects in GaAs, such as uncontrollable defects or capture centers [30]. Surface states are non-radiative in nature and can form traps in the energy structure, leading to a loss in the effective number of charge carriers penetrating the interface [31].

\section{Discussion}

In order to investigate the physical mechanism, we calculated the barrier height $\varphi_{B}$ as explained in the following. The I-V characteristic of a Schottky diode can be express as [32]:

$$
I=I_{0}\left[\exp \left(\frac{e\left(V-R_{S} I\right)}{\eta k_{B} T}\right)-1\right]
$$

where $R_{S}$ is a significant series resistance added by the semiconductor, $I_{0}$ is the reverse bias saturation current, $e$ is the electron charge, $\eta$ is the ideality factor, $k_{B}$ is the Boltzmann constant, and $T$ is the temperature. 
Considering Equation (2) and Taylor's series of the exponential function $\left(\mathrm{e}^{\mathrm{x}} \approx 1+\mathrm{x}\right)$ about the point $V=0$, we can obtain:

$$
I_{0}=\frac{\eta \frac{k_{B} T}{e} \frac{d I}{d V}}{1-R_{S} \frac{d I}{d V}}
$$

where $R_{S}$ and $\eta$ were estimated by deriving Equation (1) and considering the intercept and slope of the straight line which fits the curve:

$$
\frac{d V}{d(\ln I)}=R_{S} I+\frac{\eta k_{B} T}{e}
$$

The barrier height $\phi_{B}$ can be estimated by using the following equation [33]:

$$
\phi_{B}=\frac{k_{B} T}{e} \ln \left[\frac{A A^{*} T^{2}}{I_{0}}\right]
$$

where $A$ is the area of the device, $A^{*}$ is the Richardson's constant, that will be taken as $A^{*}=$ $156 \mathrm{~A} \mathrm{~cm}^{-2} \mathrm{~K}^{-2}$ [34], and $\mathrm{I}_{0}$ is the saturation current in reverse bias.

The calculated values of barrier height $\phi_{B}$ with respect to the light intensity for $\mathrm{Pt} / \mathrm{GaAs}$ and $\mathrm{Pt} / \mathrm{Si}$ Schottky junctions are reported in Figure 6. In our Hall sensor, $\phi_{B}$ could determine the measured voltage, which is corresponding to the previous results.

(a)

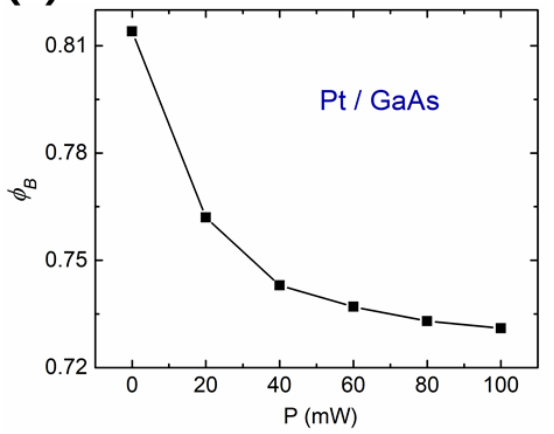

(b)

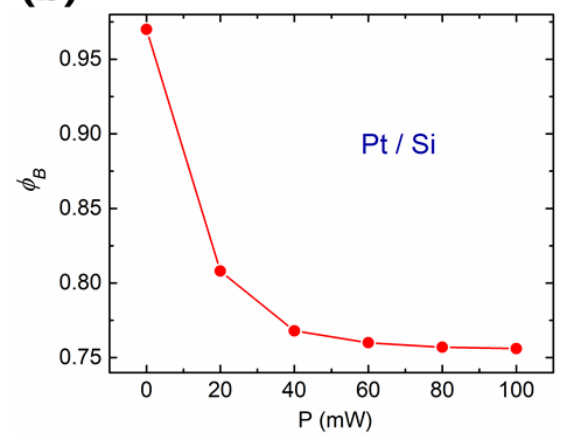

Figure 6. Barrier height as a function of the light intensity of (a) Pt/GaAs and (b) Pt/Si Schottky junctions.

From Figure 6, we found that the barrier height $\phi_{B}$ of $\mathrm{Pt} / \mathrm{GaAs}$ is lower than $\mathrm{Pt} / \mathrm{Si}$, and the change of $\phi_{B}$ is much smaller for $\mathrm{Pt} / \mathrm{GaAs}$ than for $\mathrm{Pt} / \mathrm{Si}$. It helps to demonstrate that GaAs has more surface trap states, which affect the performance of photo-induced Hall effect in our device. This issue could be alleviated by reducing the surface states through a passivation procedure in our future investigations.

Let us point out that the sensors presented here have a sensitivity of $\sim 1$ millVolt/(Watt Tesla), i.e., they would have the same sensitivity of a commercial Hall sensor if illuminated with 1 Watt, cold LED light. Besides, the sensitivity could be significantly increased by reducing the Pt thickness. Optimization of the sensors is beyond the scope of this work, in which we compare Si-based and GaAs-based sensors with same Pt thickness and light intensities.

\section{Conclusions}

In conclusion, we proposed photo-induced Hall effect in metal/semiconductor Schottky junctions as a new technique for magnetic field sensing and optical detection. Photoexcited carriers in a semiconductor drift fast to the metal, and are driven by Lorentz forces under magnetic field, then produce a transverse, open-circuit voltage at the metal edges without Joule heating. This photo-induced Hall voltage was proved to be proportional to 
the magnetic field, as well as light intensity and wavelength. The net current was zero, therefore linearity was not bias dependent. Comparison of Pt/GaAs and Pt/Si Schottky devices were carried out systematically by measuring photovoltaic I-V characteristics and bias-free, photo-induced Hall effects. The measurements of $\mathrm{Pt} / \mathrm{GaAs}$ junction are all order of magnitude lower than that of $\mathrm{Pt} / \mathrm{Si}$, and the barrier height of $\mathrm{GaAs}$ is smaller after calculations. This work not only explores the physical origins of photo-induced Schottky and Hall effects, but also reveals the relationship between the surface trap states and device performance, which provides a new approach to bias-free magnetic and optical sensing application.

Author Contributions: X.W. conceived the project. X.W. and X.S. prepared the samples. X.S. and S.C. performed the current-voltage characterizations. X.W. and X.S. carried out the Photo-induced Hall measurements. A.R. completed the model fitting. X.W., T.Z., X.S., Q.Y., J.Z., J.D. and A.R. analyzed the data and prepared the manuscript. All authors contributed to the discussion of the results. All authors have read and agreed to the published version of the manuscript.

Funding: This research was funded by the National Natural Science Foundation of China (NSFC, Grant No. 12074018), the grant from Florida Polytechnic University (Grant No. GR-2000026) and the International Research Cooperation Talent Introduction and Cultivation Project of Beijing University of Technology (Grant No. 2021C03).

Data Availability Statement: The data presented in this study are available in the article.

Conflicts of Interest: The authors declare no conflict of interest.

\section{References}

1. Hall, E.H. On a New Action of the Magnet on Electric Currents. Am. J. Math. 1879, 2, 287. [CrossRef]

2. Baibich, M.N.; Broto, J.M.; Fert, A.; Van Dau, F.N.; Petroff, F.; Etienne, P.; Creuzet, G.; Friederich, A.; Chazelas, J. Giant Magnetoresistance of (001)Fe/(001)Cr Magnetic Superlattices. Phys. Rev. Lett. 1988, 61, 2472-2475. [CrossRef] [PubMed]

3. Binasch, G.; Grünberg, P.; Saurenbach, F.; Zinn, W. Enhanced magnetoresistance in layered magnetic structures with antiferromagnetic interlayer exchange. Phys. Rev. B 1989, 39, 4828-4830. [CrossRef] [PubMed]

4. Verma, A.K.; Akkulu, P.; Padmanabhan, S.V.; Radhika, S. Automatic Condition Monitoring of Industrial Machines Using FSA-Based Hall-Effect Transducer. IEEE Sens. J. 2021, 21, 1072-1081. [CrossRef]

5. Morvic, M.; Betko, J. Planar Hall effect in Hall sensors made from InP/InGaAs heterostructure. Sens. Actuators A Phys. 2005, 120, 130-133. [CrossRef]

6. Ishibashi, K.; Okada, I. High-sensitivity Hybrid Hall Effect ICs with Thin Film Hall Elements. Sens. Mater. 2002, 14, 253-261.

7. Jiai, N. Ultra-sensitive Anomalous Hall Effect Sensors Based on Cr-doped $\mathrm{Bi}_{2} \mathrm{Te}_{3}$ Topological Insulator Thin Films. J. Phys. D Appl. Phys. 2020, 53, 505001.

8. Dowling, K.M.; Liu, T.Y. Low Offset and Noise in High Biased GaN 2DEG Hall-Effect Plates Investigated with Infrared Microscopy. J. Microelectromech. Syst. 2020, 29, 669-676. [CrossRef]

9. Nhalil, H.; Das, P.T.; Schultz, M.; Amrusi, S.; Grosz, A.; Klein, L. Thickness dependence of elliptical planar Hall effect magnetometers. Appl. Phys. Lett. 2020, 117, 262403. [CrossRef]

10. Lu, L.; Xie, H.; Luo, Z.; Muthu, N.; Chen, X.; Li, X.; Hua, Y.; Wu, Y. Frequency selectivity of spin Hall magnetoresistance sensor and its applications in eddy current testing. Appl. Phys. Lett. 2021, 118, 012403. [CrossRef]

11. Hurd, C.M. The Hall Effect in Metals and Alloys; Springer Science and Business Media LLC: New York, NY, USA, 1972.

12. Schmidt, B.; Ross, R. Position-sensitive photodetectors made with standard silicon-planar technology. Sens. Actuators 1983, 4 , 439-446. [CrossRef]

13. Li, D.; Ruotolo, A. Photo-induced Hall effect in metals. Sci. Rep. 2018, 8, 1-5. [CrossRef]

14. Blanchard, H.; De Montmollin, F.; Hubin, J.; Popovic, R. Highly sensitive Hall sensor in CMOS technology. Sens. Actuators A Phys. 2000, 82, 144-148. [CrossRef]

15. Okeil, H.; Wachutka, G. Design and Simulation of a Novel Vertical Hall Sensor With High Negative Differential Sensitivity. IEEE Magn. Lett. 2019, 10, 1-5. [CrossRef]

16. Jung, S.-Y.; Nam, K. PMSM Control Based on Edge-Field Hall Sensor Signals through ANF-PLL Processing. IEEE Trans. Ind. Electron. 2011, 58, 5121-5129. [CrossRef]

17. Bilotti, A.; Monreal, G.; Vig, R. Monolithic magnetic Hall sensor using dynamic quadrature offset cancellation. IEEE J. Solid-State Circuits 1997, 32, 829-836. [CrossRef]

18. Fasasi, T.; Ruotolo, A.; Zhao, X.; Leung, C.; Lin, K. Photo-induced anomalous Hall effect in nickel thin films. J. Magn. Magn. Mater. 2019, 485, 82-84. [CrossRef]

19. Apicella, V.; Fasasi, T.A.; Ruotolo, A. A Multilayer-Graphene/Silicon Infrared Schottky Photo-Diode. Adv. Electron. Mater. 2019, 5, 1900594. [CrossRef] 
20. Apicella, V.; Fasasi, T.A.; Wong, H.F.; Leung, D.C.; Ruotolo, A. Extending the near-infrared band-edge absorption spectrum of silicon by proximity to a 2D semiconductor. Appl. Surf. Sci. 2021, 538, 147803. [CrossRef]

21. Kim, S.Y. Generalized Schottky Anomaly. J. Korean Phys. Soc. 2014, 65, 970-972. [CrossRef]

22. Li, F. High-Temperature Current Conduction through Three Kinds of Schottky Diodes. Chin. Phys. B 2009, 11, $5029-5033$.

23. Badila, M.; Brezeanu, G.; Millán, J.; Godignon, P.; Banu, V. Silicon carbide Schottky and ohmic contact process dependence. Diam. Relat. Mater. 2002, 11, 1258-1262. [CrossRef]

24. Huang, C.-Y.; Lin, P.-T.; Cheng, H.-C.; Lo, F.-C.; Lee, P.-S.; Huang, Y.-W.; Huang, Q.-Y.; Kuo, Y.-C.; Lin, S.-W.; Liu, Y.-R. Rectified Schottky diodes that use low-cost carbon paste/InGaZnO junctions. Org. Electron. 2019, 68, 212-217. [CrossRef]

25. Ghimire, S.; Dho, J. Current-voltage characteristics and photovoltaic effect of a Au/ZnFe2O4/GaN Schottky junction. J. Phys. D Appl. Phys. 2021, 54, 095103. [CrossRef]

26. Rhoderick, E.H.; Williams, R.H. Metal-Semiconductor Contacts, 2nd ed.; Oxford University Press: Oxford, UK, 1988.

27. Quimby, R.S. Photonics and Lasers: An Introduction; Wiley-Interscience Press: Hoboken, NJ, USA, 2006.

28. Griffiths, D.J. Introduction to Electrodynamics; Pearson Prentice Hall: Upper Saddle River, NJ, USA, 1999.

29. Popović, R. Hall-effect devices. Sens. Actuators 1989, 17, 39-53. [CrossRef]

30. Hadjoub, Z.; Cheikh, K.; Zouyed, A.; Khoualdia, A.; Doghmane, A. Quantification of surface state effects in GaAs MESFETs. In IOP Conference Series: Materials Science and Engineering; IOP Publishing: Bristol, UK, 2012; Volume 28, p. 012036.

31. Chellu, A.; Koivusalo, E.; Raappana, M.; Ranta, S.; Polojärvi, V.; Tukiainen, A.; Lahtonen, K.; Saari, J.; Valden, M.; Seppänen, H.; et al. GaAs surface passivation for InAs/GaAs quantum dot based nanophotonic devices. Nanotechnology 2021, $32,130001$. [CrossRef]

32. Bartolomeo, D.A. Graphene Schottky Diodes: An Experimental Review of the Rectifying Graphene/Semiconductor HeteroJunction. Phys. Rep. 2016, 606, 1-58. [CrossRef]

33. Neamen, D.A. Semiconductor Physics and Devices, 3rd ed.; McGraw-Hill: New York, NY, USA, 2003.

34. Shimizu, T.; Okushi, H. Intrinsic Electrical Properties of Au/SrTiO 3 Schottky Junctions. J. Appl. Phys. 1999, 85, 7244. [CrossRef] 